\title{
Chain of Dirac spectrum loops of nodes in crossed magnetic and electric fields
}

\author{
V. I. Gavrilenko, ${ }^{1}$ A. A. Perov, ${ }^{2}$ A. P. Protogenov, ${ }^{2,3}$ R. V. Turkevich, ${ }^{2,3}$ and E. V. Chulkov ${ }^{4,5,6}$ \\ ${ }^{1}$ Institute for Physics of Microstructures of the RAS, 603950 Nizhny Novgorod, Russia \\ ${ }^{2}$ Nizhny Novgorod State University, 603950 Nizhny Novgorod, Russia \\ ${ }^{3}$ Institute of Applied Physics of the RAS, 603950 Nizhny Novgorod, Russia \\ ${ }^{4}$ Departamento de Física de Materiales UPV/EHU, Centro de Física de Materiales CFM - MPC and Centro Mixto CSIC-UPV/EHU, \\ 20080, San Sebastian/Donostia, Spain \\ ${ }^{5}$ Donostia International Physics Center (DIPC), 20018, San Sebastian/Donostia, Basque Country, Spain \\ ${ }^{6}$ Saint Petersburg State University, Saint Petersburg, 198504, Russia
}

(Received 21 May 2017; revised manuscript received 6 December 2017; published 16 March 2018)

\begin{abstract}
New semimetal systems along with Dirac and Weyl semimetals contain compounds, in which the energy of electron excitations vanishes not at nodes but on lines. A higher dimension of the degeneracy space changes many physical properties. We consider a chain of loops consisting of Dirac spectrum nodes in nonsymmorphic crystalline compounds placed in external mutually perpendicular magnetic and electric fields. An exact solution for the spectrum is obtained under the assumption of particle-hole symmetry. An analysis of this spectrum shows the existence of a line of critical values of the magnetic and electric fields, at which a quantum phase transition to a gapless state occurs. The use of the obtained spectrum allows also predicting a number of new oscillation and resonance effects in the field of magneto-optical phenomena.
\end{abstract}

DOI: 10.1103/PhysRevB.97.115204

\section{INTRODUCTION}

The degeneracy spaces of the electron energy spectrum in crystals, in which the valence band touches the conduction band, can be points [1,2] or lines and, in some high-dimensional cases, surfaces [3]. Since discovery of Dirac electron spectrum in graphene, and then in topological insulators, where the degeneracy space consists the nodes, the study of new electron phase states focuses on the three-dimensional case in Dirac and Weyl semimetals. Normally, these semimetals have two or more Dirac nodes, i.e., zeros of energy of bulk electron states [4]. In some cases, the spaces of occupied and unoccupied bulk states are crossed along the line, close to which the spectrum has the Dirac form. The stability of the spectrum of nontrivial phase states with respect to moderate perturbations in many of these examples is provided by the existence of topological invariants, which are defined by taking into account both the crystal symmetry and the Coulomb interaction of electrons [2]. In contrast to the first type of Dirac and Weyl semimetals, in which the spectrum is determined by linear dispersion, the dispersion law of the type-II Weyl fermions [5,6] is combined with the spectrum fragments that consist of nonrelativistic parts with quadratic electron and hole dispersions. This case is due to significantly tilted Dirac cones [6]. The electron states in the band degenerate along the lines in the three-dimensional Brillouin zone are reviewed in Ref. [7]. Exact nomenclature for the fermion spectrum with linear dispersion in systems with arbitrary dimensions and different degrees of degeneracy is discussed in the appendices of Ref. [8].

The degeneracy space structure associated with the Dirac spectrum is more complicated when the Dirac node lines are linked or form a knot. These nontrivial one-dimensional distributions of Dirac nodes are the subject of recent papers [9-15]. The systems with such esoteric properties of the degeneracy manifold need a more wide search of realizations.

The degree and type of degeneracy essentially depend on the crystalline symmetry when occupied and unoccupied bands are in contact. A detailed analysis of this dependence on symmetry of all 230 space groups was carried out in papers [16-18]. It has been shown that in crystals characterized by certain spatial symmetry and point groups, there are new fermion states with threefold, sixfold, and eightfold degrees of degeneracy at the points of contact of the band spectrum.

Crystal compounds with nodal lines of the Dirac spectrum are the subject of numerous studies [8,19-26]. Requirements of the existence of such one-dimensional manifolds from the point of view of crystalline symmetries [8,19,20,24,25] and homotopy groups [22] with or without spin-orbit interaction $[19,20,22,23]$ were considered. New compounds with nodal lines of the Dirac spectrum were also studied [8,19,21,24,25]. Besides, experimental progress [26] was recently achieved in studying the Kerr and Faraday effect to check the Berry phase contribution to quantized Kerr and Faraday rotation.

For several space groups, in the case of nonsymmorphic symmetry [20-22,24,25,27-30], the lines of nodes occur in different mutually perpendicular highly symmetric planes. These lines touch each other at points of highly symmetric axes and form a chain of double-degenerate states spreading across the entire Brillouin zone (see Fig. 1).

In some cases, a lattice of Dirac nodes in the form of network [27] can also occur in reciprocal space. The class of $X Y_{4}$ materials with such spectral features includes compounds with $X=\mathrm{Ir}$, Ta, Re; $Y=\mathrm{F}, \mathrm{Cl}, \mathrm{Br}$, I. Specific crystalline symmetries in the list of compounds [24] with nodal lines were considered in Refs. [8,19,20,24,25]. 


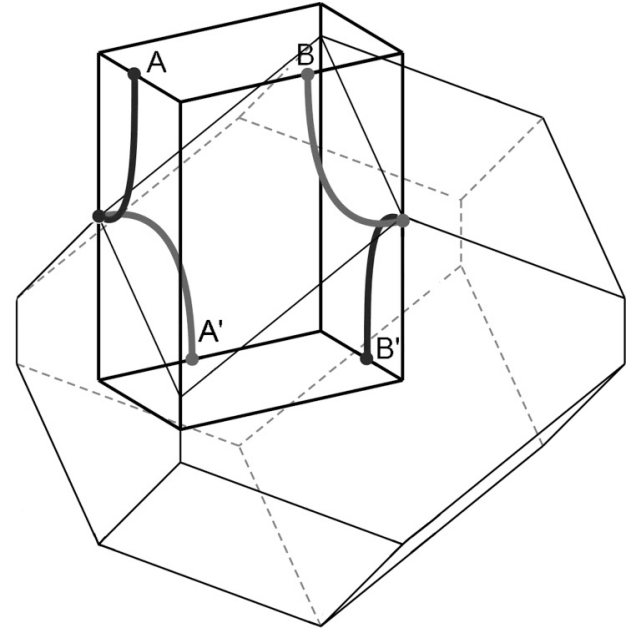

FIG. 1. A chain of Dirac nodes and its location in the Brillouin zone. The lines on the planes show different orientations of the nodal lines. Joining the points $\mathrm{A}(\mathrm{A})$ and $\mathrm{B}(\mathrm{B})$ by the nodal lines in a chiralsymmetric model forms a nodal net.

In this paper, we consider the spectrum of electron states in crystals with nonsymmorphic symmetry [30] where external constant magnetic and electric fields are imposed on the system. They will play the role of parameters that control the spectrum of Dirac states. The use of external electric and magnetic fields as handles allows tuning the band structure of nonsymmorphic topological insulators and topological phase transitions. We will examine the case of mutually perpendicular magnetic and electric fields.

\section{THE HAMILTONIAN}

In the absence of external fields, the low-energy Hamiltonian in the $\mathbf{k} \cdot \mathbf{p}$ approximation of the time-reversal invariant model containing a chain of nodes [27] is as follows

$$
\mathcal{H}=\mathcal{H}_{D}(\mathbf{k})+\mathcal{H}_{\text {pert }}=\hbar v_{F}\left[k_{x} \gamma_{1}+k_{y} \gamma_{2}+k_{z} \gamma_{3}\right]+w \gamma_{34} .
$$

This Hamiltonian has the form of the four state Hamiltonian [31,32] used for a system near a band inversion transition. In the Eq. (1), $v_{F}$ is the characteristic velocity, $\mathcal{H}_{\text {pert }}=w \gamma_{34}$, $\gamma_{34}=-\frac{i}{2}\left[\gamma_{3}, \gamma_{4}\right]$. The Dirac matrices

$$
\begin{aligned}
& \gamma_{1}=-\tau^{z} \sigma^{y}, \quad \gamma_{2}=\tau^{z} \sigma^{x}, \quad \gamma_{3}=\tau^{y} \sigma^{0}, \\
& \gamma_{4}=\tau^{x} \sigma^{0}, \quad \gamma_{5}=\tau^{z} \sigma^{z}
\end{aligned}
$$

satisfy the commutation relations $\left\{\Theta, \gamma_{a}\right\}=0,\left[\Theta, \gamma_{34}\right]=0$, where $\Theta=i \sigma^{y} \mathcal{K}$ is the time-reversal operator and $\mathcal{K}$ is the complex conjugation; $\sigma^{0}$ is the unit matrix. The Dirac matrices are chosen so that the Pauli $\tau$ matrices form $(2 \times 2)$ blocks, while $\sigma$ matrices are elements within these blocks. Thereby the four-dimensional Dirac space is presented by a set of Pauli matrices $\sigma$ for the spin degrees of freedom and by a set of Pauli matrices $\tau$ for the sublattice degrees of freedom.

Among the approaches used to study compounds with nonsymmorphic symmetry, we have chosen Hamiltonian (1) [27] for $w=0$ which, in contrast to Refs. [9-15] contains fourfold degenerate states and does not contain irrelevant terms
[23] in the product $\sigma p$. We focused on the specific form of the Hamiltonian with nonzero term $w \gamma_{34}$ in Eq. (1) as opposed to the general form [33] of perturbations since this particular case turns out to be sufficient for describing the lowenergy dynamics in systems with nodal lines. The structure of Hamiltonian (1) differs from that used in the description of Hopf-link semimetals, e.g., where the term proportional to $p_{y}$ is absent. In those cases, it was convenient to consider a reduced two-component space of states instead of a four-component space, in which Hamiltonian (1) projected onto a nodal line is determined.

The nodal chain state described by Hamiltonian (1) occurs in iridium tetrafluoride $\left(\operatorname{IrF}_{4}\right)$. The orthorhombic crystal structure of this compound belongs to the space group \#43( $F d d 2$ ). Corner-sharing octahedra of $F$ atoms enclose Ir atoms: Each Ir site of the compound $\mathrm{IrF}_{4}$ is surrounded by an octahedron of six $\mathrm{F}$ atoms, four of which are shared between the neighboring octahedra. The octahedra form a bipartite lattice as mentioned above. These two sublattices are related by an approximate chiral symmetry [27]. While filling of electron states for space group \#43 in the $\mathrm{IrF}_{4}$ compound for nonsymmorphic symmetry satisfies the special condition [27], the condition of filling of states in compounds with other space groups are different [24].

The term $\mathcal{H}_{\text {pert }}$ with $\gamma_{34}$ in Eq. (1) (as well as with $\gamma_{14}, \gamma_{24}$ ) describes zero-order perturbation in $k$, which is compatible with time-reversal symmetry and mirror symmetry $\sigma_{z}=\mathcal{I}$ 。 $C_{2 z}$. Here the operator $C_{2 z}$ is the symmetry operator of the nonsymmorphic line of Dirac nodes along the second-order rotation axis, while the operator $\mathcal{I}$ is the inversion operator. $C_{2 z}$ symmetry breaking and $\sigma_{z}$ symmetry conservation in the symmetry list that also includes the time-reversal $\Theta$ and spatial inversion $\mathcal{I}$ operators, provide the existence of nonsymmorphic lines of Dirac nodes in the mirror-invariant plane [34]. Since the bands forming nonsymmorphic nodal lines are characterized by different eigenvalues of the mirror-symmetry operator $\sigma_{z}$ (see Ref. [19]), the nodal loop is stable with respect to higher order perturbation in $k$ of Hamiltonian (1). The considered symmetry of nonsymmorphic lines of nodes is implemented in the $\beta$ phase of $\mathrm{BiO}_{2}$ [35] and in the pressurized compound $\mathrm{BaXSiO}_{4}$ [36].

The meaning of the parameter $w$ in Eq. (1) needs a special discussion. The spectrum of electron states can be easily found and is as follows:

$$
\epsilon(\mathbf{k})= \pm \sqrt{\left(\hbar v_{F} k_{z}\right)^{2}+\left(w \pm \hbar v_{F} \sqrt{k_{x}^{2}+k_{y}^{2}}\right)^{2}} .
$$

It is seen from Eq. (3) that for $k_{x}=k_{y}=0$, the last term in Eq. (1) determines the mass of excitations as they move along the axis $z$. This example shows how to define a model that contains massive excitations with time-reversal symmetry conservation in the absence of particle interaction. On the other hand, dispersion equation (3) indicates that quantization of the energy in the external magnetic field directed along the $z$ axis reduces the massive term $\left(w \pm \hbar v_{F} \sqrt{k_{x}^{2}+k_{y}^{2}}\right)$ and even makes it zero.

\section{EXTERNAL FIELDS}

Assume that the magnetic field $H$ is directed along the axis $z$, while the electric field $E$ is directed along the axis $y$. We take into account the contribution of the magnetic field to 
the dispersion relation replacing the quasimomentum components $\hbar k_{i}$ by the gauge-invariant expression $\Pi_{i}=\hbar k_{i}+e A_{i}$ containing the vector potential components $A_{i}=(-H y, 0,0)$. This specific choice of the Landau gauge does not contradict the symmetries of Hamiltonian (1), since an imposed magnetic field does not break the system symmetry: Mirror symmetry is not broken in the plane normal to the direction of the magnetic field. Symmetry with respect to a fixed-angle rotation around the magnetic field direction is also unchanged because a point group in the magnetic field contains an infinite-order rotational axis. More accurately, the point group of a crystal in a magnetic field has actually the symmetry of intersection of the crystal underlying symmetry with infinite-rotational and magnetic mirror symmetries of the magnetic field. The crystal lattice translation leads to the phase of the wave function which depends on the gauge in the magnetic field. The contribution of the electric field is determined by the term $\mathcal{H}=-e E y \hat{I}$ that supplements Eq. (1), where $e$ is the electron charge $(e>0)$ and $\hat{I}$ is the unity matrix. Taking into account the contribution of external fields, the Hamiltonian of the model is

$$
\mathcal{H}=v_{F}\left(\begin{array}{cccc}
-e \mathcal{E} y-\tilde{w} & i \Pi_{x}+\Pi_{y} & -i \hbar k_{z} & 0 \\
-i \Pi_{x}+\Pi_{y} & -e \mathcal{E} y-\tilde{w} & 0 & -i \hbar k_{z} \\
i \hbar k_{z} & 0 & -e \mathcal{E} y+\tilde{w} & -i \Pi_{x}+\Pi_{y} \\
0 & i \hbar k_{z} & i \Pi_{x}+\Pi_{y} & -e \mathcal{E} y+\tilde{w}
\end{array}\right),
$$

where $\mathcal{E}=E / v_{F}, \tilde{w}=w / v_{F}, \Pi_{x}=\hbar k_{x}-e H y, \Pi_{y}=-i \hbar \partial / \partial y$. Further, we introduce the dimensionless variable $\bar{y}=y / l_{H}$, where $l_{H}=(\hbar / e H)^{1 / 2}$ is the magnetic length. The creation operators $a^{\dagger}=\frac{1}{\sqrt{2}}(\bar{y}-\partial / \partial \bar{y})$ and annihilation one $a=\frac{1}{\sqrt{2}}(\bar{y}+\partial / \partial \bar{y})$ satisfy the commutation relation $\left[a, a^{\dagger}\right]=1$. We also introduce the parameters $\epsilon_{H}=e E l_{H} / \sqrt{2}, \epsilon_{F}=\sqrt{2} v_{F} \hbar / l_{H}$ that determine the dependence of the energy of states on the strength of external fields. Using this notation, the Hamiltonian $\mathcal{H}$ is written as

$$
\mathcal{H}=-e E l_{H}^{2} k_{x} \hat{I}-\left(\begin{array}{cccc}
\epsilon_{H}\left(a+a^{\dagger}\right)+w & i \epsilon_{F} a & i \hbar v_{F} k_{z} & 0 \\
-i \epsilon_{F} a^{\dagger} & \epsilon_{H}\left(a+a^{\dagger}\right)+w & 0 & i \hbar v_{F} k_{z} \\
-i \hbar v_{F} k_{z} & 0 & \epsilon_{H}\left(a+a^{\dagger}\right)-w & -i \epsilon_{F} a \\
0 & -i \hbar v_{F} k_{z} & i \epsilon_{F} a^{\dagger} & \epsilon_{H}\left(a+a^{\dagger}\right)-w
\end{array}\right) .
$$

This matrix has a block matrix $2 \times 2$ structure whose elements consist of $2 \times 2$ matrices $h(w)$ and $\hat{h}\left(k_{z}\right)$ and can be given as

$$
\mathcal{H}=\left(\begin{array}{cc}
h(w) & 0 \\
0 & h(-w)
\end{array}\right) \oplus\left(\begin{array}{cc}
0 & \hat{h}\left(k_{z}\right) \\
\hat{h}\left(-k_{z}\right) & 0
\end{array}\right)
$$

The particle-hole symmetry structure of Eq. (6) greatly simplifies the calculation of the spectrum. In calculations using the first term of Eq. (6), we refer to the algebraic approach applied in Ref. [37] in the two-dimensional problem of the electron spectrum in graphene in crossed fields $[37,38]$. It allows reducing matrix (5) to the diagonal form in twodimensional Fock and four-dimensional Dirac spaces. The second term in direct sum (6) after squaring leads to the relativistic dependence of the energy on $k_{z}$. This means that we can use Lorentz transformations [38] of the momentum $k_{z}$ and energy $\varepsilon$ in the frame with zero electric field $E$ to pass to the frame with $E \neq 0$.

After these calculations, in the latter frame with the magnetic field $H^{\prime}=\sqrt{1-\beta^{2}} H$ and with $\beta=E /\left(H v_{F}\right)$, we obtain the following equation for the excitation spectrum

$$
\varepsilon_{n, \pm, k_{x}}\left(k_{z} ; E\right)=-e E l_{H}^{2} k_{x} \pm \sqrt{\left(\hbar v_{F} k_{z}\left(1-E^{2} /\left(H^{2} v_{F}^{2}\right)\right)^{2}+\left(w \pm \operatorname{sgn}(n) \epsilon_{F}\left(1-E^{2} /\left(H^{2} v_{F}^{2}\right)\right)^{3 / 4} \sqrt{|n|)^{2}}\right.\right.}
$$

The form of the wave function can be obtained by generalizing the two-dimensional problem of quantum states in crossed fields to the considered three-dimensional case. The wave function takes the form of a bispinor [27], nonzero components of which are determined by the function

$$
\Psi_{n, \pm, k_{x}, k_{z}}(x, y, z)=\frac{1}{\mathcal{N}} e^{i k_{x} x+i k_{z} z} e^{-\frac{\theta}{2} \cdot \sigma_{x}}\left(\begin{array}{c}
\phi_{|n|-1}(\xi) \\
\pm i \phi_{|n|}(\xi)
\end{array}\right) .
$$

Here $\xi=\left(1-\beta^{2}\right)^{1 / 4} / l_{H} \cdot\left(y+l_{H}^{2} k_{x}+\operatorname{sgn}(n) \cdot \sqrt{2|n|} l_{H} \beta /\right.$ $\left.\left(1-\beta^{2}\right)^{1 / 4}\right)$, where $\beta=E /\left(H v_{F}\right)=\tanh \theta, \mathcal{N}$ is the normalization factor. In Eq. (8), $\phi_{|n|}(\xi)$ is the eigenfunction of the harmonic oscillator.

\section{DISCUSSION}

The term $w \gamma_{34}$ in Eq. (1) is used as perturbation. The general forms of the contributions with the terms $\gamma_{\alpha \beta}$ where $\alpha, \beta \neq$ 3,4 are discussed in Ref. [33]. The use of the perturbation term in the form of $\mathcal{H}_{\text {pert }}=b \gamma_{12}$ or in the same form with the matrix $\gamma_{23}$ or $\gamma_{31}$ leads to the following conclusions. For the first matrix, $\gamma_{12}$, we obtain the spectrum $\epsilon(\mathbf{k})=$ $\pm \sqrt{\hbar^{2} v_{F}^{2}\left(k_{x}^{2}+k_{y}^{2}\right)+\left(\hbar v_{F} k_{z} \pm b\right)^{2}}$ of Weyl semimetals. For the second (third) matrix, the indices of the axes $z$ and $x$ ( $z$ and $y$ ) interchange. These spectra take place in systems where spatial inversion symmetry is conserved and time-reversal 
symmetry is broken. If $\mathcal{H}_{\text {pert }}=b \gamma_{45}$, then $\epsilon(\mathbf{k})= \pm \hbar v_{F} k \pm b$. In this case, spatial inversion symmetry is broken and timereversal symmetry is conserved. The specific symmetry of the nonsymmorphic line system based on the $w \gamma_{34}$ breaking term in the Hamiltonian can be implemented under strain due to applied pressure as we mentioned in Sec. II.

It is useful to compare the asymptotic cases that result from Eq. (7) with known answers. In the absence of an electric field for $w=0$, we have a well-known expression for the relativistic particle spectrum in the external magnetic field $[39,40]$. If the massive term $w \neq 0$ and the electric field $\mathbf{E}=0$, we obtain the result of Ref. [27]. In the absence of a massive term $w=0$ and an electric field $\mathbf{E} \neq 0$ in the plane $k_{z}=0$, we come back to the results of Refs. [37,38]. As in Refs. [37,38], in the threedimensional case, we deal with the collapse of Landau levels due to the factor $\alpha=1-E^{2} /\left(H^{2} v_{F}^{2}\right)$ and the corresponding modification of the wave function. The argument $\xi$ of the function $\phi$ in Eq. (8) also describes the shift of the Gaussian function center of a harmonic oscillator due to the electric field.

Stability of the nontrivial phase is guaranteed by the $\mathbb{Z}_{2-}$ valued topological invariant [27] $z_{2}=\frac{1}{\pi} \oint d \mathbf{k A}(\mathbf{k}) \bmod 2$, where $\mathbf{A}(\mathbf{k})=i \sum_{n}<u_{n}(\mathbf{k}) \mid \nabla_{\mathbf{k}} u_{n}(\mathbf{k}>$ is the Berry connection. The constraint of the Chern integer by $\bmod 2$ is, on the one hand, a specific feature of the considered three-dimensional system in comparison with the standard Dirac semimetal state. On the other hand, the topological invariant $z_{2}$ is similar to the $\mathbb{Z}_{2}$-valued topological invariant [41-43] which was used to classify the boundary states in 3D topological insulators. Transition of the quantum phase to the trivial phase state with $z_{2}=0$ is usually associated with a vanishing gap in the energy spectrum. The value $z_{2}=1$ of the invariant $z_{2}$ corresponds to the topologically nontrivial state [27].

In the considered two-parameter $(E, H)$ case, the equation for the effective gap is

$$
\Delta_{\text {eff }}=w \mp \operatorname{sgn}(n) \epsilon_{F}\left(1-E^{2} /\left(H^{2} v_{F}^{2}\right)\right)^{3 / 4} \sqrt{|n|} .
$$

This function is zero in the plane $(E, H)$ along the line described by the equation

$$
E(H)=v_{F} H\left(1-\left(\frac{H_{n}}{H}\right)^{2 / 3}\right)^{1 / 2} .
$$

Here $H_{n}=w^{2} /\left(2 \hbar e v_{F}^{2}|n|\right)$ (see Ref. [27]). For different numbers of the Landau level $n$, the arising set of critical lines of the quantum phase transition is limited by $E=v_{F} H$, as the magnetic field increases for $H>H_{1}$.

The external electric field not only modifies the wave function and spectrum. It can also disturb the ground state by creation of particle-hole pairs. This kind of local dielectric breakdown in graphene [38] has a specific length and a Landau index dependence. The considered $3 D$ system does not differ from the $2 D$ case in this respect.

Exact solution (7) of the problem can be used in various applications, e.g., for calculating the conductivity $\sigma(\omega, q)$. Analysis of the resonance denominator $\varepsilon_{n, p_{z}}+\hbar \omega=\varepsilon_{n^{\prime}, p_{z}+\hbar q}$ of susceptibility and the numerator that determines the infeasibility and feasibility conditions of a quantum transition from the state with quantum numbers $\left(n, p_{z}\right)$ to the state with the numbers $\left(n^{\prime}, p_{z}+\hbar q\right)$ enables one to solve several problems.

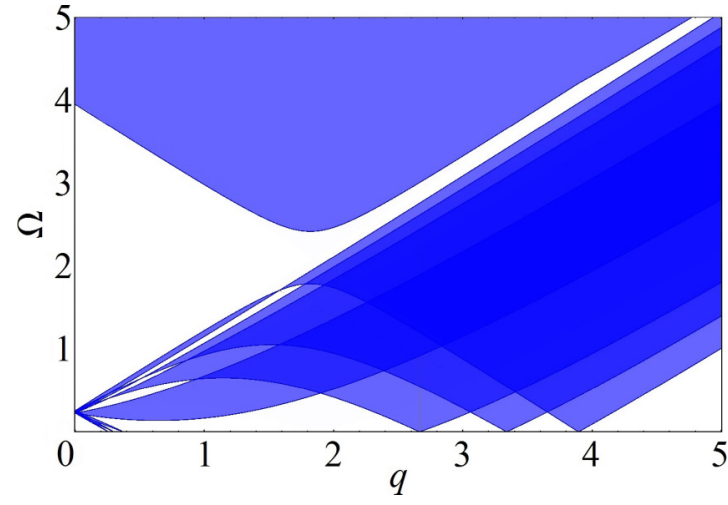

FIG. 2. Landau damping regions for left-hand polarized waves in the case of four occupied Landau levels and $\Omega \simeq 2 \mathcal{E}_{F}=4.2, q \simeq$ $2 k_{F}=4.2, w / E_{0}=0.2$.

This approach allows constructing Landau damping regions at arbitrary frequencies $\omega$ and for the wave vectors q. In these regions, the real part of conductivity determines the Landau damping of collective modes. Besides, additional transparency windows can exist within the Landau damping regions, in which new collective modes can propagate. This approach for the parabolic spectrum was used in Ref. [44] to find the dispersion law of right-hand polarized $\left(n^{\prime}=n-1\right)$ collective electromagnetic excitations. In the considered model, we found the Landau damping regions for left-hand polarized waves $\left(n^{\prime}=n+1\right)$. They are plotted in Fig. 1 . When constructing the Landau damping regions, we used the following dimensionless variables

$$
\begin{aligned}
& \Omega=\hbar \omega / E_{0}, q=\hbar v_{F} q_{z} / E_{0}, \mathcal{E}_{F}=E_{F} / E_{0}, \\
& k_{F}=p_{F} v_{F} / E_{0}, E_{0}=\epsilon_{F}\left(1-E^{2} /\left(H^{2} v_{F}^{2}\right)\right)^{3 / 4} .
\end{aligned}
$$

The difference of the considered case from the nonrelativistic electron spectrum in a quantizing magnetic field used in Ref. [44] is in taking into account the final gap $w$, the relativistic nature of the problem, and the use of the cyclotron frequency $\tilde{\omega}_{c}=\sqrt{2}\left(v_{F} / l_{H}\right)\left(1-\beta^{2}\right)^{3 / 4}$ modified by the electric field. It is clear from Fig. 2 that the factor $\left(1-\beta^{2}\right)^{3 / 4}$ in this equation will also modify the universal collisionless width $\tilde{\omega}_{c}$ of the cyclotron resonance peak and the spectrum of the left-hand circularly polarized collective modes in the region $0 \leqslant \Omega \leqslant$ $1,0 \leqslant q \leqslant 1$. This means that a variation of the electric field strength $E$ enables one to control the resonance phenomena in the medium. These magnetospectroscopic effects and the regions of Landau collisionless damping of longitudinal and transverse polarized collective excitations will be analyzed separately in Ref. [45].

Magneto-optical effects have been studied theoretically and experimentally in a variety of papers. It should be emphasized that the information provided by Faraday spectroscopy of semiconductor structures is detailed. The main advantage of this method is its higher sensitivity compared to that of direct detection of spectrum splitting. The frequency dependences of Kerr and Faraday angles can be effectively used in experimental studies of complex structures of spectra in the magnetic field. Note that the magneto-optical angles obtained in Ref. [26] are comparable to those for interband transitions 
in ferromagnetics or metal-alloyed semiconductors [46-48] and strained nonmagnetic semiconductors based on gallium arsenide [49]; the magneto-optical angles are also comparable to the Kerr angle in some layered metal structures [50] or organic molecular semiconductors [51]. Anisotropy of the energy spectrum should essentially affect the Kerr rotation character in the studied materials. The properties of surface states of carriers are important for Kerr rotation. Therefore, the Kerr effect can differently manifest itself at normal incidence of an electromagnetic wave on various nonsymmorphic crystal faces. Study of Faraday rotation in film structures is also of current interest [26].

In the discussion of the Landau damping and the spectrum of collective modes, we assume that temperature dependence of the distribution function is absent and the lifetime of electron excitations is the largest of all time scales [44]. When taking into account the final temperature $T$ and the final lifetime $\tau$ of the charge carriers, these collective phenomena can occur if $k_{B} T \ll E_{0}\left(E_{0} / E_{F}\right)$ and $\hbar / \tau \ll E_{0}\left(E_{0} / E_{F}\right)$ because the right-hand side of these conditions is the difference $E_{n+1}\left(k_{F n}\right)-E_{n}\left(k_{F n}\right)=\sqrt{E_{F}^{2}+E_{0}^{2}}-$ $E_{F} \approx E_{0}\left(E_{0} / 2 E_{F}\right)$ between the Landau levels for $k_{F n}=$ $\sqrt{E_{F}^{2}-E_{0}^{2}|n|} / \hbar v_{F}$. Here $k_{B}$ is the Boltzmann constant and $E_{F}$ is the Fermi energy. These conditions can be provided at helium temperatures and in magnetic fields of the order of $40 \mathrm{~T}$.

In summary, we obtained the analytic solution for the spectrum of electron excitations in the materials containing a chain of loops that consists of Dirac spectrum nodes. The case where these nonsymmorphic crystalline compounds are placed in crossed external magnetic and electric fields is considered. The spectrum analysis indicates the existence of lines of critical values of electric and magnetic fields, for which a quantum phase transition to a gapless state is possible.

After submission of the present paper, we became aware of the results of Ref. [52], where Eq. (7) for $w=0$ was obtained by a different method. Electron excitation spectrum can also be controlled by crystal lattice deformation. An example of such deformation by pseudogauge fields is given in this paper.

\section{ACKNOWLEDGMENTS}

This work was supported in part by Project No. 3.2637.2017/4.6 (A.A.P.) of the Ministry of Education and Science of the Russian Federation, by Project No. 15.61.202.2015 of Saint Petersburg State University (E.V.Ch.), and Grant No. IT-756-13 of the Basque Country University (E.V.Ch.). One of the authors (A.P.P.) is grateful to the International Physics Center in San Sebastian for hospitality.
[1] C. Herring, Phys. Rev. 52, 365 (1937).

[2] G. E. Volovik, The Universe in a Helium Droplet (Oxford University Press, New York, 2003).

[3] B. Lian and S.-C. Zhang, Phys. Rev. B 94, 041105 (2016).

[4] X. Wan, A. M. Turner, A. Vishwanath, and S. Y. Savrasov, Phys. Rev. B 83, 205101 (2011).

[5] A. A. Soluyanov, D. Gresch, Z. Wang, Q.-S. Wu, M. Troyer, X. Dai, and B. A. Bernevig, Nature 527, 495 (2015).

[6] G. E. Volovik and K. Zhang, J. Low Temp. Phys. 189, 276 (2017).

[7] C. Fang, L. Lu, J. Liu, and L. Fu, Nat. Phys. 12, 936 (2016).

[8] B. J. Wieder, B. Bradlyn, Z. Wang, J. Cano, Y. Kim, H.-S. D. Kim, A. M. Rappe, C. L. Kane, and B. A. Bernevig, arXiv:1705.01617.

[9] Z. Yan, R. Bi, H. Shen, L. Lu, S.-C. Zhang, and Z. Wang, Phys. Rev. B 96, 041103(R) (2017).

[10] X.-Q. Sun, B. Lian, and S.-C. Zhang, Phys. Rev. Lett. 119, 147001 (2017).

[11] B. Lian, C. Vafa, F. Vafa, and S.-C. Zhang, Phys. Rev. B 95, 094512 (2017).

[12] W. Chen, H.-Z. Lu, and J.-M. Hou, Phys. Rev. B 96, 041102 (2017).

[13] M. Ezawa, Phys. Rev. B 96, 041202(R) (2017).

[14] P.-Y. Chang and C.-H. Yee, Phys. Rev. B 96, 081114 (2017).

[15] G. Chang, S.-Y. Xu, X. Zhou, S.-M. Huang, B. Singh, B. Wang, I. Belopolski, J. Yin, S. Zhang, A. Bansil, H. Lin, and M. Z. Hasan, Phys. Rev. Lett. 119, 156401 (2017).

[16] B. Bradlyn, J. Cano, Z. Wang, M. G. Vergniory, C. Felser, R. J. Cava, and B. A. Bernevig, Science 353, aaf5037 (2016).

[17] J.-Z. Ma, C.-J. Yi, B. Q. Lv, Z. J. Wang, S.-M. Nie, L. Wang, L.-Y. Kong, Y.-B. Huang, P. Richard, H.-M. Weng, B. A. Bernevig, Y.-G. Shi, T. Qian, and H. Ding, Sci. Adv. 3, e1602415 (2017).
[18] B. J. Wieder, Y. Kim, A. M. Rappe, and C. L. Kane, Phys. Rev. Lett. 116, 186402 (2016).

[19] Y. Kim, B. J. Wieder, C. L. Kane, and A. M. Rappe, Phys. Rev. Lett. 115, 036806 (2015).

[20] B. J. Wieder and C. L. Kane, Phys. Rev. B 94, 155108 (2016).

[21] J.-M. Carter, V. V. Shankar, M. A. Zeb, and H.-Y. Kee, Phys. Rev. B 85, 115105 (2012).

[22] C. Fang, Y. Chen, H.-Y. Kee, and L. Fu, Phys. Rev. B 92 081201(R) (2015).

[23] K. Mullen, B. Uchoa, and D. T. Glatzhofer, Phys. Rev. Lett. 115 , 026403 (2015).

[24] B. Bradlyn, L. Elcoro, J. Cano, M. G. Vergniory, Z. Wang, C. Felser, M. I. Aroyo, and B. A. Bernevig, Nature 547, 298 (2017).

[25] R. Takahashi, M. Hirayama, and S. Murakami, Phys. Rev. B 96, 155206 (2017).

[26] L. Wu, M. Salehi, N. Koirala, J. Moon, S. Oh, and N. P. Armitage, Science 354, 1124 (2016).

[27] T. Bzdusek, Q.-S. Wu, A. Ruegg, M. Sigrist, and A. A Soluyanov, Nature 538, 75 (2016).

[28] Y. Chen, H.-S. Kim, and H.-Y. Kee, Phys. Rev. B 93, 155140 (2016).

[29] R. Chen, H. C. Po, J. B. Neaton, and A. Vishwanath, Nat. Phys. 14, 55 (2017).

[30] Y. X. Zhao and A. P. Schnyder, Phys. Rev. B 94, 195109 (2016).

[31] Z. Gao, M. Hua, H. Zhang, and X. Zhang, Phys. Rev. B 93, 205109 (2016).

[32] B.-J. Yang and N. Nagaosa, Nat. Comm. 5, 4898 (2014).

[33] A. Westström and T. Ojanen, Phys. Rev. X 7, 041026 (2017). 
[34] A. A. Burkov, M. D. Hook, and L. Balents, Phys. Rev. B 84, 235126 (2012).

[35] S. M. Young, S. Zaheer, J. C. Y. Teo, C. L. Kane, E. J. Mele, and A. M. Rappe, Phys. Rev. Lett. 108, 140405 (2012).

[36] J. A. Steinberg, S. M. Young, S. Zaheer, C. L. Kane, E. J. Mele, and A. M. Rappe, Phys. Rev. Lett. 112, 036403 (2014).

[37] N. M. R. Peres and E. V. Castro, J. Phys. Cond. Mat. 19, 406231 (2007).

[38] V. Lukose, R. Shankar, and G. Baskaran, Phys. Rev. Lett. 98, 116802 (2007).

[39] A. H. MacDonald, Phys. Rev. B 28, 2235 (1983).

[40] M. Orlita, D. M. Basko, M. S. Zholudev, F. Teppe, W. Knap, V. I. Gavrilenko, N. N. Mikhailov, S. A. Dvoretskii, P. Neugebauer, C. Faugeras, A.-L. Barra, G. Martinez, and M. Potemski, Nat. Phys. 10, 233 (2014).

[41] L. Fu and C. L. Kane, Phys. Rev. B 74, 195312 (2006).

[42] T. Fukui and Y. Hatsugai, J. Phys. Soc. Jpn. 76, 053702 (2007).

[43] D. Niesner, Th. Fauster, S. V. Ereemev, T. V. Menshchikova, A. P. Protogenov, E. V. Chulkov, O. E. Tereshchenko, K. A.
Kokh, O. Alekperov, A. Nadjafov, and N. Mamedov, Phys. Rev. B 86, 205403 (2012).

[44] V. Ya. Demikhovskii and A. P. Protogenov, JETP Lett. 11, 591 (1970).

[45] R. V. Turkevich, A. P. Protogenov, P. M. Echenique, and E. V. Chulkov (unpublished).

[46] A. Stroppa, S. Picozzi, A. Continenza, M. Y. Kim, and A. J. Freeman, Phys. Rev. B 77, 035208 (2008).

[47] M.-H. Kim, G. Acbas, M.-H. Yang et al., Phys. Rev. B 75, 214416 (2007).

[48] A. Winter, H. Pascher, M. Hofmayer et al., Rev. Adv. Mater. Sci. 20, 92 (2009).

[49] Y. K. Kato, R. C. Myers, A. C. Gossard, and D. D. Awschalom, Phys. Rev. Lett. 93, 176601 (2004).

[50] C. Etz, A. Vernes, L. Szunyogh, and P. Weinberger, Phys. Rev. B 77, 064420 (2008).

[51] M. Fronk, B. Brauer, J. Kortus, O. G. Schmidt, D. R. T. Zahn, and G. Salvan, Phys. Rev. B 79, 235305 (2009).

[52] V. Arjona, E. V. Castro, and M. A. H. Vozmediano, Phys. Rev. B 96, 081110 (2017). 